\title{
The Reasonable and Conscious Understanding System of reality Under Uncertainty
}

\author{
B. Khayut, L. Fabri, M. Avikhana \\ Intelligent Decisions Technologies Systems at IDTS \\ Israel
}

Received: March 27, 2020. Revised: June 30, 2020. Accepted: July 9, 2020. Published: July 13, 2020.

\begin{abstract}
The modern autonomous Expert and Statistical Systems of Artificial Intelligence (AI) cannot continuously, independently and consciously think, learn and develop. This is happening because the models, methods and technologies of their processing in these systems cannot synchronously actualized (trained), function, independently, systemically, situationally, continuously, accurately and on their own in the conditions unpredictability, uncertainty of changing situations and lack of data, information and knowledge about the objects during the process of their continuous perception from the fuzzy environmental reality. Consequently, the need arises to create self-learning, self-developing and self-organized computational intelligent systems that continuously perceive and process changing data, information and knowledge in their changing, uncertainty and previously unknown situation in the surrounding reality. To solve the above problems and to create a system of General AI, we offer the new concept of creating a Computational Intelligent System of a Reasonable and Conscious Understanding of reality under uncertainty through of developed by us following models, methods and technologies of: a) perception the reality of environment, b) self-developing memory, c) situational control of data, information, knowledge, objects, models and processes, d) presentation, generalization and explanation of knowledge, e) fuzzy inference, f) decision making, g) reasoning and thinking, h) cognition, and h) Dialog Control in communication with human, robots and systems through of the intelligent interface, which integrating this functionality into a coherent Reasonable and Conscious Understanding System of reality Under Uncertainty.
\end{abstract}

Keywords-reasonable, conscious, understanding, system.

\section{INTRODUCTION}

$\mathrm{T}$ HIS article suggests a new concept, method, model and technology of creation the Reasonable and Conscious Understanding System of the Reality, functioning Under Uncertainty (RCUSRUU) as a plug-in in Intelligent Complex Adaptive Systems (ICAS). The functionality of RCUSRUU is implemented with of the developed by us of the selfdeveloping computational methods of modeling of the: a) Perception of languages, images, sounds and their objects of the changing reality, b) Identification, classification, structuration, categorization, transformation, memorization, actualization of the mentioned semantic objects and their data, information and knowledge in the self-organized Thesaurus (Knowledge Base), where these objects are interconnected each to other in Neural Network c) Systems Situational Fuzzy Control [1], [2] of data, information, knowledge, objects, models and processes, d) Fuzzy Inference, e) Decision making, Reasoning and Systems Thinking, g) Cognition, and h) communication with human, robots and systems through of the Intelligent User Interface (IUI).

This concept, method, model and technology of implementation of the RCUSRUU were applied the principles of the Systems Approach [3] and Situational Control [4], as well as the main aspects of Fuzzy Logic [5], [6], Linguistics, Cybernetics and Computer Science at whole, which allowed this system to be self-organized and operate computationally, intellectually, independently (without participation of the human intellect), systemically, continuously and in the advance unknown situations.

At the core of the modern Expert AI (EAI) and Statistical AI (SAI) systems is rigidly pre-defined in Expert or Statistical models, that programmers constantly reprogram when the logic of the business model is changed. The changes in process of control of organizational unit entail changes in the script menu dialog. This leads to a continuous redesign and reprogram of the EAI and SAI systems. Moreover, these models do not self-changing in real time situationally, independently, continuously and synchronously in line with situational changing business models of surrounding reality and do not always predict the sustainable result with high accuracy due to the use in them of probability statistical methods. Consequently, the probabilistic statistical models of EAI and SAI systems are unreliable due to the unpredictability of obtaining their accurate sustainable results in continuous real time conditions and the uncertainty of the state of data, information and knowledge about changing objects of the changing surrounding reality. In these circumstances, it becomes impossible, as example, to make relevant decisions in situations, which vary in real time, because the decisions taken no longer correspond to the changed state of the model 
in the changed situation, which violates the principle of Situational Control [4] and of continuity of functioning of the models in EAI and SAI systems due to interruption of the process of their functioning for training their models by reprogramming them in conditions of changing the busines logic of environmental objects.

To avoid such a discrepancy, it becomes necessary to use open models that satisfies the following principle of Situational Control [4] in the design of the RCUSRUU:

- All information about the data, information and knowledge (about the organizational unit) may be communicated to the control system as a set of phrases of Natural Language (NL), images, sounds and others.

- Control model is fundamentally should be open and never ends by the creation of the final formal model.

- Description of the process of data management (information, knowledge) is possible only in the form of natural phrases and / or on another language, images and sounds and others.

The advantage of the proposed RCUSRUU includes the:

- Modelling and situational fuzzy control of data, information and knowledge should be implemented an automatic fuzzy inference, decision making, reasoning, thinking, mind, awareness, consciousness and other offered by us methods to find on their basis the correct, accurate, timely and adequate decision, considering a current situation and impact of fuzzy environment on the state of the models.

- Use of those decisions, criteria and objectives for providing of planning and control of the business processes in the fuzzy environment.

- Processes of extracting and converting of images, concepts, meanings of various natural languages and subject areas, and serializing them into the bases of data, information and knowledge.

- Use of these bases for realization of the proposed Concept, multi-lingual dialog control, generalization and explanation of knowledge, and other processes in the RCUSRUU.

- Use of properties of a) atomicity of data, b) connectedness of information, c) figurativeness, associativity and activity of knowledge for their integration and aggregation.

- Use of methods of reasoning, systems thinking, psychology and others to obtain the decision of high quality and precision under uncertainty.

For clarity of the terminology used, we will give some definitions [7], [8] of the concepts, related to the understanding of the problem, considered in this article.

Data are organized in the memory and are perceived by the person or machine as facts, numbers, words, symbols, lines and other items of information. They are not related to each other and are found in texts, pictures and in other objects of reality.

Information is a group of related data, organized in the memory that respond to the questions of "who", "what", "where", "when" and others.

Knowledge is the image of the subject model or region, which are extracted from information and are organized in memory with the properties be interpreted, structured, associated, transformed, updated, activated, analyzed, deduced, stored and so on, answering on the questions "why" and "how to", satisfying the criteria and objectives of their existence in the reality.

Modeling decisions is defined as a process of construction of a new conceptual situation and a state of controlled units (fuzzy data, information, knowledge, inference and others), which meets the criteria and purposes of the information system in fuzzy environment.

Planning decisions is defined as a use of modeling results to create a sequence of alternative decisions that will match to the situation and the state of information system in the subsequent stages of management of the organizational unit.

Decision-making is defined as a process of modeling fuzzy inference [10] for selection the relevant decision from limited number of alternative decisions, obtained during the planning decisions.

Fuzzy Control is the process, which uses the results of modeling, planning and decision-making in fuzzy environment, in order to implement a control action on the data, information and knowledge with the goal of moving the control system into the new state, which corresponds to a specified condition.

Uncertainty defines the current states of knowledge of the object and its fuzzy environment in an extreme and unforesee situation, where their properties, structure, origin, nature, method for producing and sequence of processing of this object in the environment are not known beforehand and necessary to implement the intelligent analysis in real time for computating of the mentioned characteristics of the object, its state and state of its environment in the arisen situation.

The Reasoning [8] is the process of using existing knowledge to draw conclusions, make predictions, construct generalizations and explanations, using deductive, inductive and other methods for gaining new knowledge and parcels using the Situational Knowledge Control [2], Modeling of Fuzzy Inference [10] and a Decision making [11] under uncertainty.

The Systemic Thinking [12] is a process of: a) generalization of results, obtained by the group of active processes of Reasoning [9], based on methods of applying of Systems Approach [3] and Situational Control [4] and principles under uncertainty and Fuzzy Environment, and b) detecting and predicting the behavior of the system by modeling of the process of Systems Thinking [12], actualization his current state, enhancing its stability, viability, integrity, and providing its interaction with other its surrounding systems.

The Perception is the process of obtaining a knowledge from a data and information, including the properties of environment and its objects with the use of process of Systems Thinking for the purpose of Cognition a data and an information, generalization, actualization and memorization (storing) their state in their repositories in continuous real time.

The Cognition is concidered to be a continuosly in real time process of Perception of objects in their reality, implemented 
by the process of Systems Thinking to the current unstructured and related previously stored data, information and knowledge, using the proper hardware and software, related to the sense of of sight, hearing, taste, touch, and more, with the objective to generate new data, information and knowledge. The method to be applied in this case is the Systems Thinking process, which involves and combines the processes of Perception, Cognition, Situational Fuzzy Control and also a Computational Artificial Nervous System [8] (conductor of decisions), Sensory systems of perception of objects of reality and system of assessing of the surounding impacts on these processes and systems.

Consciousness is considered by us as a property of Systems of General Artificial Intelligence (SGAI), which is modeled through of process of understanding one's own and environmental states, as well as actions, that take them to a new state under conditions of uncertainty and of the current situation.

Under the Dialog Control we mean the process of presenting partners of common commands (questions) each to other using languages, images and sounds and others through its IUIs and providing by system of targeted actions (issuing replies), which are relevant to the topic of the dialogue and the situations in which it occurs.

Artificial Intelligence (AI) system is understood by us as a knowledge-based system, which is a secure electronic autonomous system, and which a) runs on the operating system b) is connected to the Internet, d) performs native or cloud-based applications, e) analyzes the collected data [13], information and knowledge, and e) performs the functions of a human-computer interface for solving problems in a fuzzy environment.

Subject area understood by us as branch of knowledge and technologies, where the organizational units are functioning.

Environment is the surrounding reality, consisting of organizational units, information systems, robots, human and so on, which interact with each other under the influence of the environment.

The Machine Consciousness (MC) [14] is explored in AI, Cognitive Robotics, Philosophy and others by solving problems of the computational Mind, Consciousness and mental state.

We believe, that an effective solution to the problem of Computational Consciousness lies in the systemic applying of the main achievements of Fuzzy Logic [5], [6], Situational Control [4], linguistics, Cognitive Psychology, Computer Science and other sciences at whole.

Our analysis of the state of scientific research in the field of creation of Systems of SGAI showed that the directions and methods of their implementation are mainly related with the introduction in them of the principles of systemic and situational control, the continuity of their functioning in conditions of uncertainty, adaptability of their models to environmental changes and the level of their functionality. In this context, we will conduct a brief of comparative analysis of our approach and the approaches of other authors to create the SGAI

In [15] is considered the possibility of the computational synthesis of artificial consciousness, which include a number of the following principles: The Brain is a State Machine, Inner Neuron Partitioning, Conscious and Unconsciousness States, Perceptual Learning and Memory, Prediction, the Awareness of oneself, Representation of Meaning, Learning Utterances, Learning Language, Will, Instinct and Emotion. However in this paper, not applied the principles of a Systems Approach, Situational Control, Fuzzy Logic and other sciences in general, which does not allow the models of computational consciousness to be open (self-changing) in the conditions of uncertainty and unexpected changes in objects of Fuzzy Environment. Also, are not considered the models and methods for implementing computational Fuzzy Inference, Systems Thinking, and other functions of the computational Brain (Fig. 3), without which, in our opinion, its computational consciousness is impossible.

The article [16] describes the model of the relationship between self-awareness and consciousness in the neural network scheme developed, used in experiments with robots to determine the ability to perceive, feel and be aware of events of objects that occurred or existed in the environment. The model of self-consciousness and its relationship with consciousness was studied and presented through of the use of a neural network and three robots. Were investigated the reactions of the robots in the conditions of independence of one of them, without of their thinking under uncertainty. The approach to solving the problem of computational consciousness in our paper differs from [16], that the models of computational consciousness in [16] are hard predefined and retrained periodically (reprogrammed) by the human intellect and are not changed independently, continuously, situationally and synchronously in accordance with changing of fuzzy environment and the objects in it. Also, these robots are not able to independently and systemically think logically and make decisions under uncertainty, which means they do not know, what they are doing. This means that their consciousness is not relevant and is absent between the intervals of reprogramming.

Based on our analysis of the above works and others, we came to the conclusion, that along with the existing achievements in the field of Machine Consciousness, its effective functioning in conditions of uncertainty of a changing external environment is impossible without using the principles of a Systems Approach, Situational Control, Fuzzy Logic, Computer Science and others sciences at whole.

Consequently, the traditional methods of Systems Thinking are characterized by: a) lack of situational principle of knowledge control, b) pre-known scenarios, which are clearly defined in advance, c) no variability of structures and relationships in the knowledge, d) limited activity of knowledge, e) inability to update of knowledge in accordance with the changing environment, and others.

Given these and other shortcomings we propose a systemic and linguistic approach to Reasonable and Consciousness Understanding of changing objects in reality under uncertainty and changing them in Fuzzy Environment, using the principles of Situational Control and Fuzzy Logic theories, General AI, 
Linguistics, Knowledge based technology and others.

In this laboratory study, we practically applied some theoretical results from the theories of Fuzzy Logic, Situational Control, Computational Linguistics, Relational Databases and Computer Science at whole and have generalized the concept of the linguistic variable of fuzzy logic, expanding it with psychological, pragmatic aspects along with the semantic and reasonable and conscious aspects of Natural Languages Processing (NLP). These linguistic aspects and generalization together with the aspects of Situational Control, Relational Database and Computer Science at whole were applied in computational laboratory experiments to develop the structure of the Neural Network of fuzzy semantic frames, the mentioned Thesaurus, Knowledge Base and systems of the Fuzzy Situational Control of Data, Information, Knowledge, objects, models, NLP and full understanding and consciousness of the meanings of objects of
Thinking are characterized by: a) lack of situational principle of knowledge control, b) pre-known scenarios, which are clearly defined in advance, c) no variability of structures and relationships in the knowledge, d) limited activity of knowledge, e) inability to update of knowledge in accordance with the changing environment, and others.

Toward to SGAI, we offer and apply the developed by us conceptual computational model, method, a generalized algorithm and a system of modelling systemic machine consciousness under uncertainty, using of our self-developed neural memory network, containing integrated and embedded each to other semantic fuzzy frames (Table 1, Fig. 1), models, methods of Situational Fuzzy Control of data, information, knowledge and objects [2], Fuzzy Inference [10], Decision Making [11], Knowledge Representation [9], Knowledge Generalization [17], Knowledge Explanation, Reasoning [9], Systems Thinking [12], Cognition [9], IUI [7], Computational

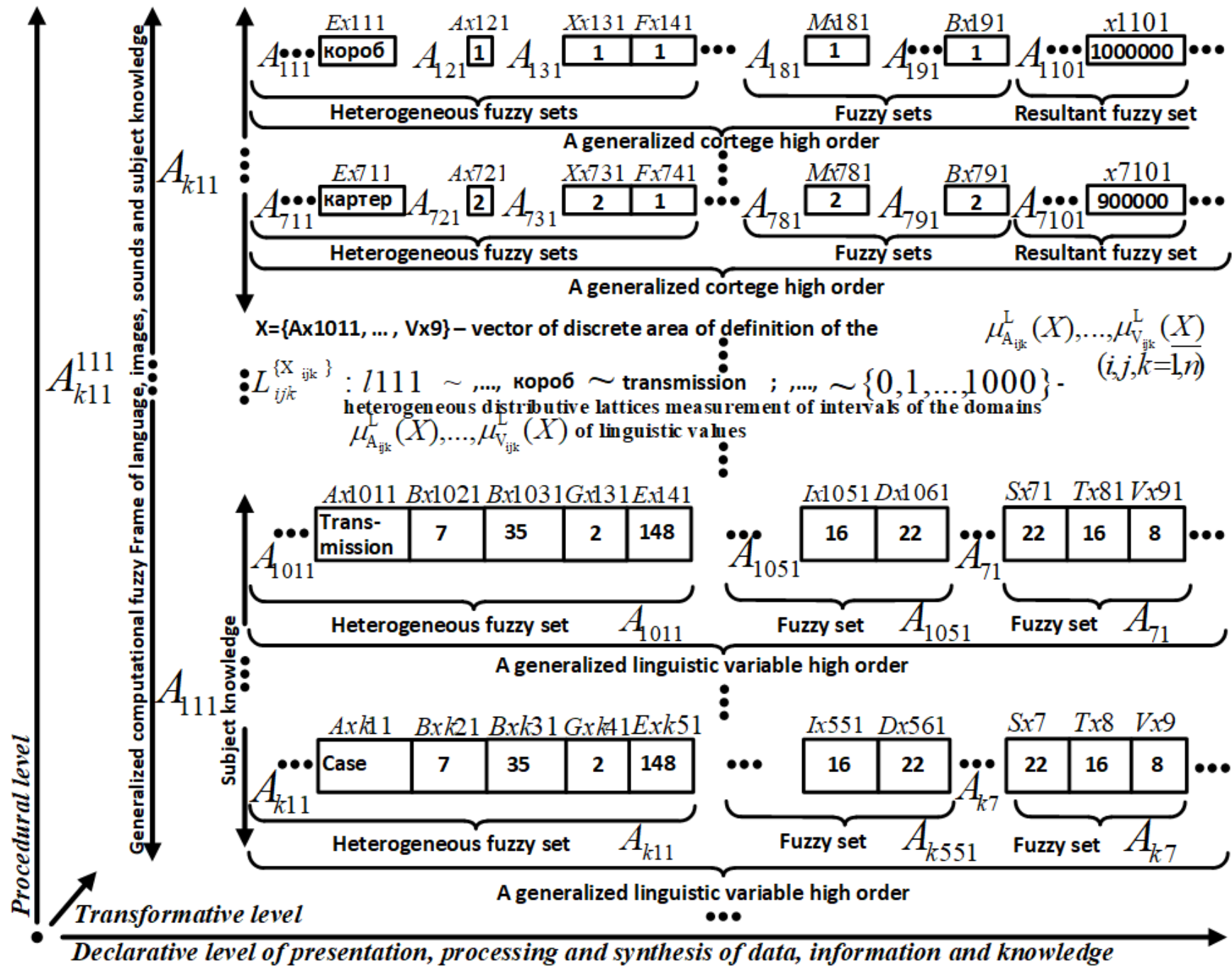

Fig. 1. The Neural Network of the Semantic Fuzzy Frames of the language, visual, sound subject area objects in the Systemic Consciousness Memory Model.

reality, that expressed by words, sentences and texts of natural languages in various subject areas and their subdomains. Consequently, the traditional methods of Systems
Systemic Mind [8], Awareness and Full Understanding of the reality[18]. The modelling of these processes is implemented by way of continuously processing languages, sounds and 
visual objects, their structures and states, perceived from the changing reality under uncertainty.

\section{The Model Of The Memory Of The Systemic COMPUTATIONAL CONSCIOUSNESS}

\section{A. The Computational Consciousness Memory Model}

The memory model of Systemic Computational Consciousness is represented by a continuously selfdeveloping neural network of semantic fuzzy frames of nested and integrated into each other, and their elementary data, information and knowledge, organized as interconnected sets of generated linguistic variables, fuzzy sets, values of which reflect the, syntactical (grammatical, morphological and lexical), semantic, mental and other categories, properties, features, concepts and essences in subject areas (Table 1, Fig. $1)$.

Table 1. The Language and subject area models of Data, Information and Knowledge in Systemic Consciousness Memory.

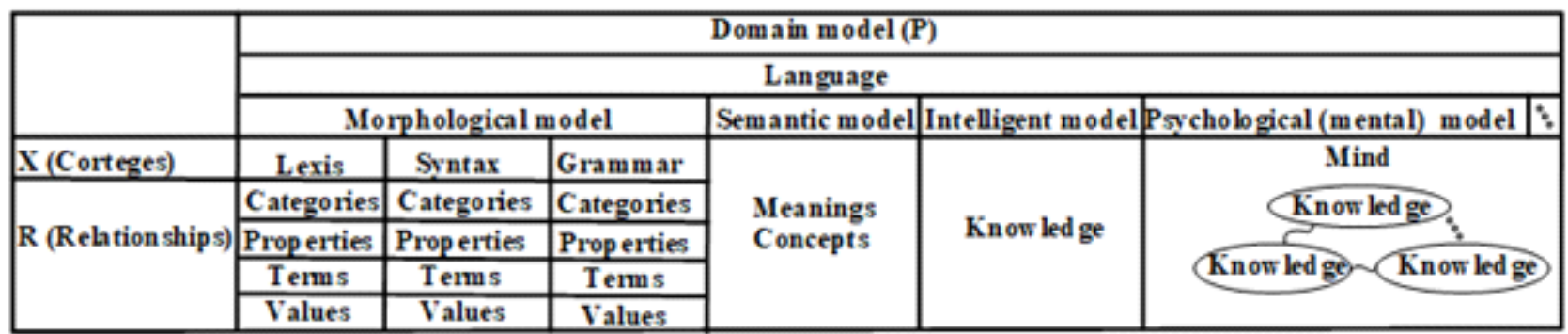

The Data, Information, Knowledge and Essences are extracted from texts, images and sounds by detection their thematic (Subject Areas), types of terms, values, properties, categories, concepts and meanings, that are processed using Analyzers and stored in the Neural Network of the Semantic Fuzzy Frames in the Memory using of the developed SelfOrganized Computational Thesaurus (Knowledge Base) (Fig. 2).

The Self-developing Thesaurus is working continuously, accumulate knowledge from the perceived and detected establishing semantic relationships between detected objects, their processing, actualizing and storage.

The Synthesis of the Computational Consciousness (Fig. 2) is implemented on the basis of the results of the Syntactic, Semantic and Intelligent Analyzers, which, respectively, perceive, process linguistic, visual and sounds objects, store them in Thesaurus, which is used by Analyzers for processing and synthesis of Computational Consciousness.

The Fig. 1. Displays the Fuzzy Logic's generated Linguistic Variables, Fuzzy Sets and their values for representing of semantic fuzzy frames of Data, Information and Knowledge [2] of the language, visual and sound objects and their extracted properties from the reality.

The Fig. 1. presents a sample of a generalized scheme of fuzzy sets of linguistic and subject area data, information, and knowledge used to support the modeling process of computational consciousness, where for the subject area data, information and knowledge, for example the:

$\mathrm{X}(\mathrm{Ax} 1011, \mathrm{Bx} 1021, \ldots, \mathrm{Vx} 91, \ldots)$ is a vector of identification of discrete values areas of definition of the membership functions $\mu_{A}^{L}(X), \ldots, \mu_{V}^{L}(X)$ for objects of languages, images, sounds and others,

$\mathrm{L}$ ('Transmission', '7', .., ' 8 ', ...) is a vector of values, accepted from heterogeneous distribution lattices L, from where the membership functions $\mu_{A}^{L}(X), \ldots, \mu_{V}^{L}(X)$ gets values for objects of languages, images, sounds and others.

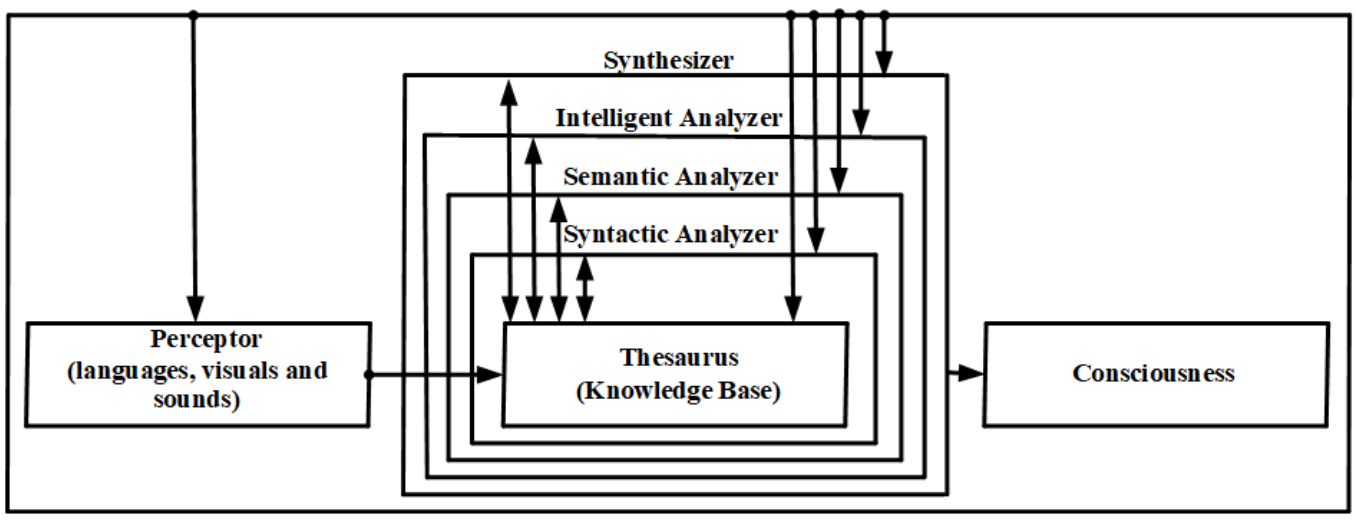

Fig. 2. Synthesis of Systemic Computational Consciousness Under Uncertainty.

objects of the reality, and thus self-learns, implementing automatic coding of the objects, their classification, clustering unification, elimination of duplication, integration,
Each of mentioned vectors contain groups of heterogeneous fuzzy sets A, which together represent a generalized high order tuple. These tuples are representing a generalized group 
of a fuzzy subject of fuzzy linguistic and subject data, information and knowledge identifying the values of the integrated fuzzy linguistic and subject area categories, properties, terms and their fuzzy semantic meanings. These tuples are representing a generalized group of a fuzzy subset of fuzzy linguistic and subject data, information and knowledge identifying the values of the integrated fuzzy linguistic and subject categories, properties, terms and their fuzzy semantic meanings. These groups of fuzzy subsets are integrated into generalized and integrated computational frames of languages, images, sounds and so on, and represent fuzzy knowledge in considered subject areas. These integrated fuzzy knowledge, information and data is declaratively presented, procedurally processed, transformed (Fig. 1) and displayed in current situations, described by languages, images and sounds in the internal representation of consciousness and synthesized into external representations that are understandable to people and systems. This process is controlled by the situational control system of the Computational Mind system [8] (Fig. 3).

The Memory Model represents the self-organized Neural Network semantically interconnected, integrated and nested into each other objects of fuzzy logic and their detailed properties, categories and features related to heterogeneous subject areas and reflecting data, metadata, information and knowledge at the declarative, procedural and transformative levels (Fig. 1) of their processing using the above-mentioned models, methods and systems. The considered Memory Model reflects Morphological, Semantic, Pragmatic, Psychological (mental) and their aspects of languages, images and sounds.

\section{The COMPutational Systemic COnsciousness Model}

\section{A. The Model of Situational Fuzzy Control of}

Computational Systemic Consciousness Modeling process

The model of Situational Fuzzy Control of Computational Systemic Consciousness Modeling process is represented in the systemic computational consciousness model in the form (1), [8].

$$
\mu=\left\langle A_{n}^{S}, K_{n}^{S}, F_{n}^{S},\left(S_{i}: Q_{i} \stackrel{x u_{R} w}{\Rightarrow} Q_{1}: I_{i}\right) \cdot\left(\mu_{R}^{Y}\left(\mu_{R}^{E}\left(\mu_{R}^{L}\left(\mu_{R}^{Z}\left(\mu_{R}^{D}\left(\mu_{R}^{M}\left(\mu_{R}^{T}\left(\mu_{R}^{H}\left(\mu_{R}^{C}\left(\mu_{R}^{0}\right)\right)\right)\right)\right)\right)\right)\right)\right)>\right.\right.
$$

The (2) and (3) are representing, respectively, the sub model of Memory and aimed Processing, and sub model of Situational Fuzzy Control in model of the Computational Systemic Consciousness Modeling (1) by using linguistic and theme data, information, knowledge, objects, models and processes under uncertainty of them in the Fuzzy Environment,

$$
\begin{aligned}
& M=\left(\mathbf{A}_{\mathbf{n}}^{\mathbf{s}}, \mathbf{K}_{\mathbf{n}}^{\mathbf{s},}, \mathbf{F}_{\mathbf{n}}^{\mathbf{s}}, \mathbf{M}_{\mathbf{B}}^{\mathbf{R}}(\mathbf{X}), \mathbf{S}\right) \\
& \mathbf{S}=\left(\mathbf{S}_{\mathbf{i}}: \mathbf{Q}_{\mathbf{j}} \underset{\mathbf{u}, \mathbf{x}, \mathbf{w}}{\Longrightarrow} \mathbf{Q}_{\mathbf{l}} ; \mathbf{I}_{\mathbf{i}}\right)
\end{aligned}
$$

where, respectively, in (2) and (3):

$A_{n}^{S}$ - Data, Information and Knowledge Memory Model, which represents of a mental Concept, represented by sets of generalized linguistic variables (generalized situations), which constituents dictionary entries of linguistic, thematic and other types of objects, defined in generalized thesauri, as segments of accumulation in them of these objects (Fig. 1).

$K_{\mathrm{n}}^{g}$ - Data, Information and Knowledge systemic processing Model of concepts $A_{n}^{a}$ using of inhomogeneous linguistic, thematic and other data, information, knowledge, objects, models and processes, organized and processed on declarative, procedural and transformative levels of fuzzy modeling of knowledge, containing the relationships of concepts, defined as mental computational compositional mapping rules (5) of modeling of linguistic, thematic, mental and other data, information, knowledge, objects and processes.

$$
F_{N}^{S}=u(x, w)
$$

- represents a Model of Objectives of Systemic Situational Computational Consciousness modeling under uncertainty. This model defines in (1) the objectives of modeling and situational fuzzy control of linguistic, thematic, mental and other data, information, knowledge, objects, models and processes.

$M_{B}^{R}(X)$ - The Computational Systemic Consciousness Fuzzy Modeling system, realized by the Computational Control, Inference, Decisions Making, Knowledge Discovery, Knowledge Representation, Explanation, Reasoning, Systems Thinking, Cognition, Machine Learning, Systemic Mind and Awareness.

$\mathrm{S}_{\mathrm{i}}-$ full $\mathrm{Q}_{\mathrm{j}}-$ current and $\mathrm{Q}_{\mathrm{l}}-$ new situations of state of data, information, knowledge and other objects in process of modeling of Computational Systemic Situational Consciousness.

$I_{i}$ - presents a generated compositional mapping rule (5), which converts the model and the RCUSRUU into new mental state in situation,

$x$ - are definite Fuzzy control actions to manage fuzzy sets $\mathrm{A}$ and $\mathrm{B}$.

$w$ - are indefinite Fuzzy control actions to manage fuzzy sets $\mathrm{A}$ and $\mathrm{B}$.

$u-$ is a resultant targeted situational control action

(decision) (4), which is expressed on language thought and is generated by the system of Situational Fuzzy Control of Computational Systemic Consciousness to bring the RCUSRUU from the previous state to a new state in the arisen situation.

\section{The Systemic Situational COMPUTATIONAL CONSCIOUSNESS MODELING UNDER UNCERTAINTY}

\section{A. The Model of Systemic Situational Computational Consciousness Modeling process}

According to sub models (2), (3) and Fig. 3 the model (1) presents the process of the Situational Fuzzy Control of Computational Consciousness Modeling in Computational Mind System [8], where: 
$\mu_{n}^{Y}-$ a Cyber-physical module in Computational Mind $I_{i}$ - the generated Consciousness system's rules of impacts System, objects of data, information, knowledge, that are used to

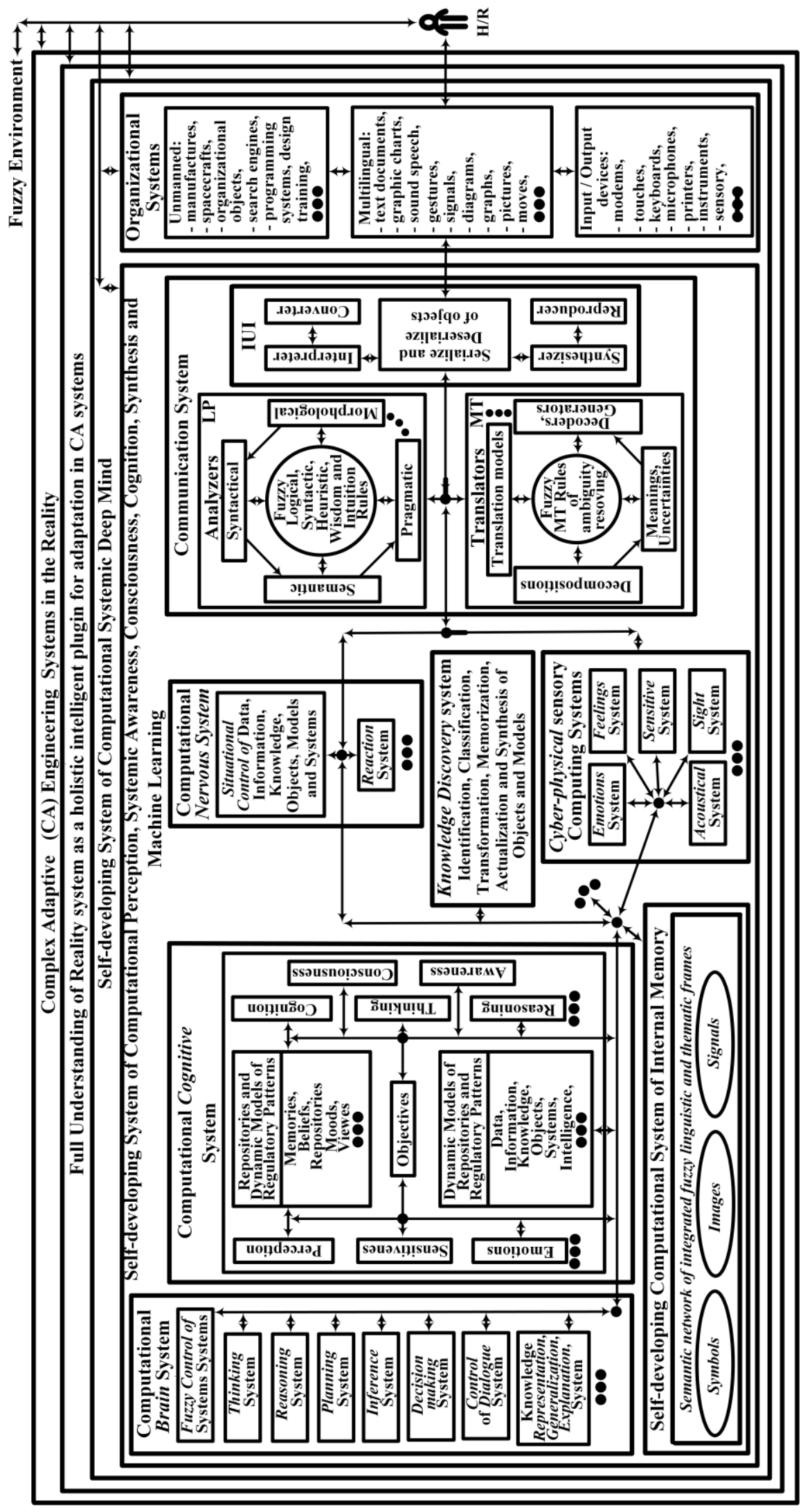

Fig. 3. The process of Computational Consciousness Modeling in the Computational Systemic Mind system. 
actualize the situation $\mathrm{S}_{\mathrm{i}}$ after as the state of the Situational Consciousness Fuzzy Control system had been shifted from the previous $\mathrm{Q}_{\mathrm{j}}$ to the new state $\mathrm{Q}_{\mathrm{l}} \cdot \mu_{\mathrm{R}}^{\mathrm{F}}$ - a module of generation of the target of compositional rules and Situational Fuzzy Control of actions of actualization, coordination of communication processes,

$\mu_{\mathrm{R}}^{\mathrm{L}}$ - a module of computational Systemic Fuzzy Logic Inference,

$\mu_{\mathrm{R}}^{\mathrm{Z}} \quad$ - a module of computational Presentation, Generalization and Explanation of Knowledge

$\mu_{\mathrm{R}}^{\mathrm{D}}$ - a module of computational Fuzzy Control of Dialog and Communication with users and systems,

$\mu_{\mathrm{R}}^{\mathrm{M}}$ - a module of computational Decision making and Planning decisions,

$\mu_{\mathrm{R}}^{T}$ - a module of computational mental Systemic Reasoning,

$\mu_{\mathrm{R}}^{\mathrm{H}}$ - a module of computational mental Systemic Thinking,

$\mu_{\mathrm{R}}^{\mathrm{C}}$ - a module of computational mental Systemic Cognition,

$\mu_{\mathrm{R}}^{0}$ - a module of computational mental Systemic Consciousness.

In Fig. 4 represented a process of modeling the Situational Systemic Computational Consciousness using the mentioned models (2), (3) and the model (1) at whole in the

Computational Brain System, which is a component of the Computational Mind System (Fig. 3).

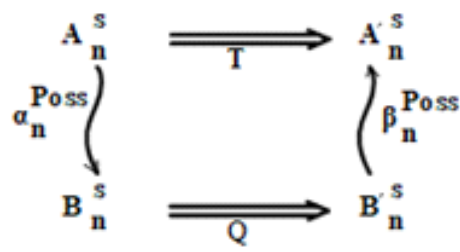

Fig. 4. Diagram of Modeling of the Situational Systemic Computational Consciousness.

The process of Modeling of Situational Systemic Computational Consciousness activates of embedded in each other computational processes of modeling of knowledge, fuzzy logic inference, decision making, planning of decisions, fuzzy control, dialogue control, reasoning, systems thinking, perception, cognition and consciousness. These processes are implemented with using of the process of systemic situational control of data, information, knowledge and objects, where the result of the previous process of modeling is the same for all the processes, with the difference, that each objective function (4) determines the objective of modeling according of RCUSRUU's functional requirements during its functioning.

The Situational Systemic Computational Consciousness uses the Properties (Modules, Processes, mentioned in the model (1)) of Computational artificial Brain, generated by work of its procedures. The result of this work is a vector of values of internal and external output fuzzy variables, obtained by using a new vector of the values of the input fuzzy variables, which used by these procedures to bring the model and the RCUSRUU in the new state (in accordance with the situation at the current time) by means of method of determination of fuzzy matching in fuzzy modeling of consciousness (Fig. 1) by using of objectives (4), compositional mapping rules (7), constraints, associations and measures of opportunities, presented in (8).

According of the Situational Control model (3), the processes in the Situational Systemic Computational Consciousness model (Fig. 4) means the following:

$\alpha_{n}^{\text {Poss }}, \beta_{n}^{\text {Poss }}-$ are, respectively, mappings, defined by matrices of verbal and numerical estimates of fuzzy relationship functions $M_{B}^{R}(X), M_{A}^{R}(X)$ and relationships $R_{B}^{X}$, $R_{A}^{X}$ (Fig. 1.).

$A_{n}^{s}, A_{n}^{I S}$ - are current States of the Consciousness models (respectively, before and after their actualization).

$B_{n}^{s}, B_{n}^{v s}-$ are mapped States of the Consciousness models (respectively, before and after their actualization), related to the States $A_{n}^{s}, A_{n}^{s g}$.

$\mathrm{T}, \mathrm{Q}$ - are transformation operators of the Situational Fuzzy Controlled Consciousness models $B_{n}^{s}$ and $B_{n}^{s s}$ from Current their States to their New States.

The Computational Consciousness is defined in [8] as" a property, created as a result of computational activity of the computational Brain, which is a self-assessment of the degree of truth of the perceived objects of reality".

The Fig. 3. presents the process of Computational Consciousness Modeling through of the Computational Systemic Deep Mind system, which is described in [8].

According to [8] (Fig. 3.) the process of the Computational Consciousness Modeling is carried out using of the following Computational Systemic Deep Mind system's functions of the: a) Computational Brain System, b) System of Computational Cognition, c) Computational Nervous system, d) Knowledge Discovery system, and e) Cyber-physical sensory Computational System.

The Computational Brain and Cognitive systems include and integrate in itself the Computational Consciousness subsystem, which together with Computational Nervous System, Knowledge Discovery Cyber-physical sensory and Communication systems are subsystems of the Selfdeveloping System of Computational Perception, Systemic Awareness, Consciousness, Cognition and ML systems, which are embedded as subsystems into Self-developing System of Computational Systemic Deep Mind.

All processes of the mentioned subsystems and systems are controlled by the Situational Fuzzy Control System of Data, Information, Knowledge, Objects, Models and Systems, which together with the Reaction Systems are embedded into Computational Nervous System, which situationally controlled by the Computational Brain System and all the mentioned systems interact interconnectedly within the framework of the System of Computational Mind.

Thus, the processes of Computational Consciousness are supported by all intelligent processes of System of Computational Systemic Deep Mind and versa.

\section{B. Modeling of Situational Systemic Computational Consciousness}

The technology of modeling of Computational Consciousness is implemented by way of using the developed methods of 
Situational Control, Systems Approach and procedures of fuzzy logic Inference, Decision Making, Systems Thinking and Cognition, which are realized by Module of Knowledge $K_{n}^{s}$ in (3), where frames $\varphi_{1}^{q}: U_{k}^{m}<->V_{k}^{\mathbb{D}}$ are selected by $\varphi_{1}^{q}$ as fuzzy matching operation in procedure of Systemic Computational Consciousness process using fuzzy sets $U_{k}^{m}$ and $V_{k}^{\mathrm{l}}$, and compositional mapping rules (5):

$$
\mu_{b}^{q}(X)=\mu_{a}^{q}(X) * \varphi_{0}^{q}
$$

where:

$\mu_{b}^{q}(X), \mu_{\pi}^{q}(X)$ are respectively, the resultant and initial membership functions in the considered generalized fuzzy relation $R_{k}^{X}$;

$\mathrm{X}=\left\{x_{k}^{q}\right\}$ is a vector of the resultant and initial membership functions.

$a$ and $b$ - are the inhomogeneous multi-dimensional fuzzy sets.

- is a sign of the compositional mapping rules.

$l$ - are the inhomogeneous distributive lattices of measurement intervals of the domains of definition of considered membership functions,

$q$ - determine the declarative, procedural, transformation and other levels of representation and processing of the Data, Information and Knowledge in Memory,

$$
k, l=(1, \mathrm{n}) \text {. }
$$

Under the fuzzy matching of fuzzy sets we mean the action, performed by procedures with the frame $\Phi_{L_{j k}}^{q}: U_{k}^{m} \leftrightarrow V_{k}^{l}$, which generate the matching of base fuzzy sets $U_{k}^{m}$ and $V_{k}^{\mathbb{1}}$ in RCUSRUU. This matching of base fuzzy sets is implemented by using of compositional mapping rules

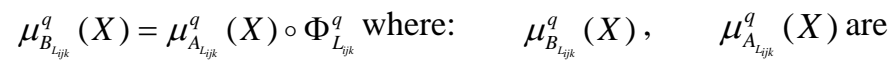
respectively, the resultant and initial membership functions in the considered generalized fuzzy relation $R_{i j k}^{X_{i j k}}, X=\left\{x_{i j k}\right\}$ is a vector of the discrete domain of definition of resultant and initial membership functions, ${ }^{\circ}$ - is a sign of the computational mapping, $\Phi_{L_{j k}}^{q}$ is a fuzzy matching in the procedure of Situational Systemic Computational Consciousness, $A_{L_{j k}}, B_{L_{j k}}$ - are the in heterogeneous multi-dimensional fuzzy sets, $L_{i j k}$ are the heterogeneous distributive lattices of measurement intervals of the domains of definitions of considered membership functions, $q$ - determine the mentioned above levels, respectively, on sublevels of $R X$-codes, USK Universal Semantic Code and $S F$ - Semantic Frames, $l, k, m=$ $(1, n)$.

The degree of conformity of the numerical, verbal, mental and other estimates of concepts $x$ from $X$ are determinate by fuzzy sets $A^{\alpha}=\left\{x, \mu^{A}(x) \geq \alpha^{A}\right\}$ and by relations $R^{\alpha}=\left\{x, \mu^{R}(x) \geq \alpha^{R}\right\}$ on all of the mentioned levels and sublevels of modeling of Situational Systemic Computational Consciousness and establishing the values of $\alpha$ - levels for
$A^{\alpha}$ and $R^{\alpha}$ (in the certain and various distributive lattices $L$, "nearest" to the top of the curves $\mu^{A}$ and $\mu^{R}$ at each of considered levels of modeling of Situational Systemic Computational Consciousness), which leads (using $\mu_{R}^{O}$ in (1) ) to unit all of "tops" $\alpha$ - slices and to the information of the resulting fuzzy set of unique (generalized) and relevant data, information, knowledge and objects, characterizing the reached level of Situational Systemic Computational Consciousness.

\section{The Model of Computational Mapping Rules for Modeling Situational Systemic Computational Consciousness}

According of the provided above analysis the (6),

$$
\mu_{b}^{q}(X)=v\left(\wedge \mu_{a}^{q}(X) \wedge \text { Poss }\left(a_{k}^{q} / a_{k}^{q}\right)\right.
$$

determines the model of compositional mapping rules (5) in process of creation properties of Situational Systemic Computational Consciousness, implemented with using measures of opportunities Poss $\left(a_{k}^{q} / a_{k}^{q}\right)$ on $q$ levels of modeling data, information and knowledge.

The result of modeling of Situational Systemic Computational Consciousness is a multidimensional generalized rule - relationship of (a generalized computational frame) $R_{k}^{X}$ with a domain of values $\mu_{v}^{\varphi}:\left\{x_{k}^{q}\right\}$ $\rightarrow L_{k}^{x}$. This rule is the relation, that determines a distributed chain of parcels $\mu_{F}^{\varphi}$ at levels of internal and external representations, modeling and processing knowledge and each of these parcels is forming a group of (computing) values.

The technology of modeling of Situational Systemic Computational Consciousness is implemented by way of using the developed methods of application of Situational Control, System Approach and procedures of Fuzzy Logic Inference, Decision Making, Systems Thinking, Reasoning, Awareness, Consciousness and Cognition, which are realized by Module of Knowledge $K_{n}^{s}$ in (2).

The computational mapping rules model (7) determines the

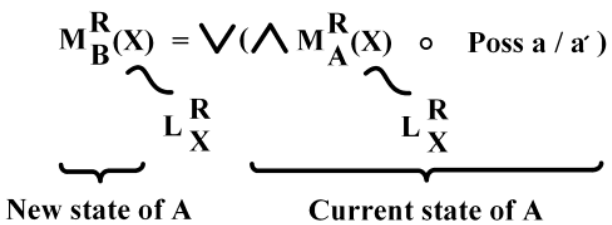

compositional mapping rules of modeling of Situational Systemic Computational Consciousness using the measures of opportunities Poss a / $\mathrm{a}^{\prime}$ (7) on declarative, procedural and transformative levels of modeling data, information, knowledge and objects (Fig. 1.), where:

$\mathrm{A}$ and B are Fuzzy Sets, R represents the generalized relationships,

$\mathrm{X}$ is a vector (cortege) of Terms, Values, Properties, Categories, Concepts, Meanings and Knowledge (Table 1),

$\mathrm{L}$ are various distributive lattices of the $\alpha$-values, of the membership functions $M_{A}^{R}:\{x\} \rightarrow L_{M}^{R}$ and $M_{B}^{R}:\{x\} \rightarrow L_{X}^{R}$.

The computer representation of one of the computational 
rules (7) of resolving lexical ambiguity in process of modeling Systemic Computational Consciousness for language objects processing is given by (7) and Fig. 5. (a, b). by human, devices, systems and processes for subsequent processing.
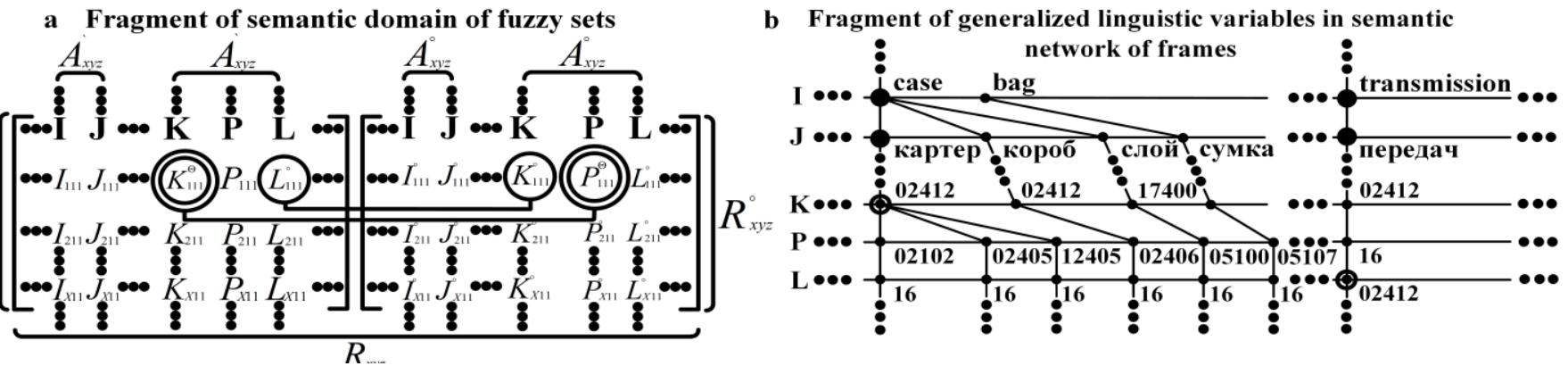

Fig. 5. (a) External; b) Internal levels of modeling of Systemic Computational Consciousness.

This process operates at the internal (machine) and external (human symbolic, sound, visual and others) levels of representation and modeling of Computational Consciousness, using multidimensional generalized matrices, obtained from a model of a semantic network of computational frames, organized in the memory of the computational system of Consciousness (Fig 1). The presented in Fig. 1 the declarative, procedural, transformational and other levels of integrated processing the subject, linguistic, sound and visual data, information, knowledge and objects determine the dimension of the above-mentioned matrices. The Transformational level of processing provides the transformation of the machine level of the presentation of data, information, knowledge and objects into their external representation and vice versa, through of the procedures of modeling computational consciousness, which in turn use the procedures of the computational Brain system, which is a component of the computational Mind system (Fig. 3).

For example, for the language objects the rule (8) is implemented in the computational model (2) by the Module $K_{\mathrm{n}}^{s}$, as follows:

$$
\begin{gathered}
\left.\operatorname{IF}\left(\left(K_{x y z}^{*}=P_{x y z}^{*}\right) \text { AND } L_{x y z}^{\infty}=K_{x y z}^{*}\right)\right) \text { THEN } \\
\left.\left(\left(I_{x y z}=I_{x y z}^{\infty}\right) \text { AND } l_{x y z}=l_{x y z}^{\infty}\right)\right)
\end{gathered}
$$

if two words are included in sentence in both languages and the code of right compatibility of word, located to the left of a group of two words in another language is equal to conceptual code of the word, located to the right of a group of two words in the output language and the code of left compatibility of word, located to the right of a group of two words in the output language is equal to conceptual code of word, located to the left of a group of two words in input language, then these pairs of words of input and output languages correspond and are identical each to other at the level of conceptual disambiguation in mapping process.

Thus, the result of work of the modules (1), (2), (7) and (8) is a relationship, which comprises the relevant syntactical, grammatical, logical, semantic, psychological and pragmatic data, information, which correspond to the situation at the current moment of resolution of the ambiguity and maps the objects of reality on language of thought in the internal and external levels of their representations in memory, perceptible
D. The General Algorithm of Situational Systemic Computational Consciousness Modeling

The General Algorithm of the Situational Systemic Computational Consciousness Modeling process in process of Content synthesis from English Texts is presented on Fig. 6. by the following four main processes (Fig. 2.):

The Perception process:

- Perceives the speech texts, split them on sentences, chunks, clauses, phrases and their words.

- Extracts the syntax (grammar, morphological and lexical) categories, properties, terms and values (Fig. 1.), their structures and relationships for each sentence and its chunks, clauses and phrases.

The Morphological process:

- Extracts words' morphological categories, properties and values.

The Semantic process:

- Extracts meanings and concepts from each word and sentence at whole.

- Extracts syntax, grammar, lexical and semantic structures for each sentence from texts.

- Identifies, classifies, clusters and stores all perceived and extracted syntactical (grammatical, morphological, lexical), semantical categories, properties, terms, values, meanings, concepts in neural semantic network Memory, including Thesaurus (Knowledge Base) and other Repositories.

The Intelligent process is activated and controlled by the Computational Brain and Cognitive systems [8]

(Fig. 3), which:

- Organizes Situational Control and Actualization of Data, Information, and Knowledge, Models, Processes and their objects.

- Provides Fuzzy Inference, Decision Making, Reasoning, Systems Thinking, Mind, Awareness and Consciousness.

- Provides Representation, Generalization and Explanation of Knowledge.

- Provides the Computational Content synthesis and its Full Understanding.The General Stages of Situational Systemic Consciousness Modeling. 


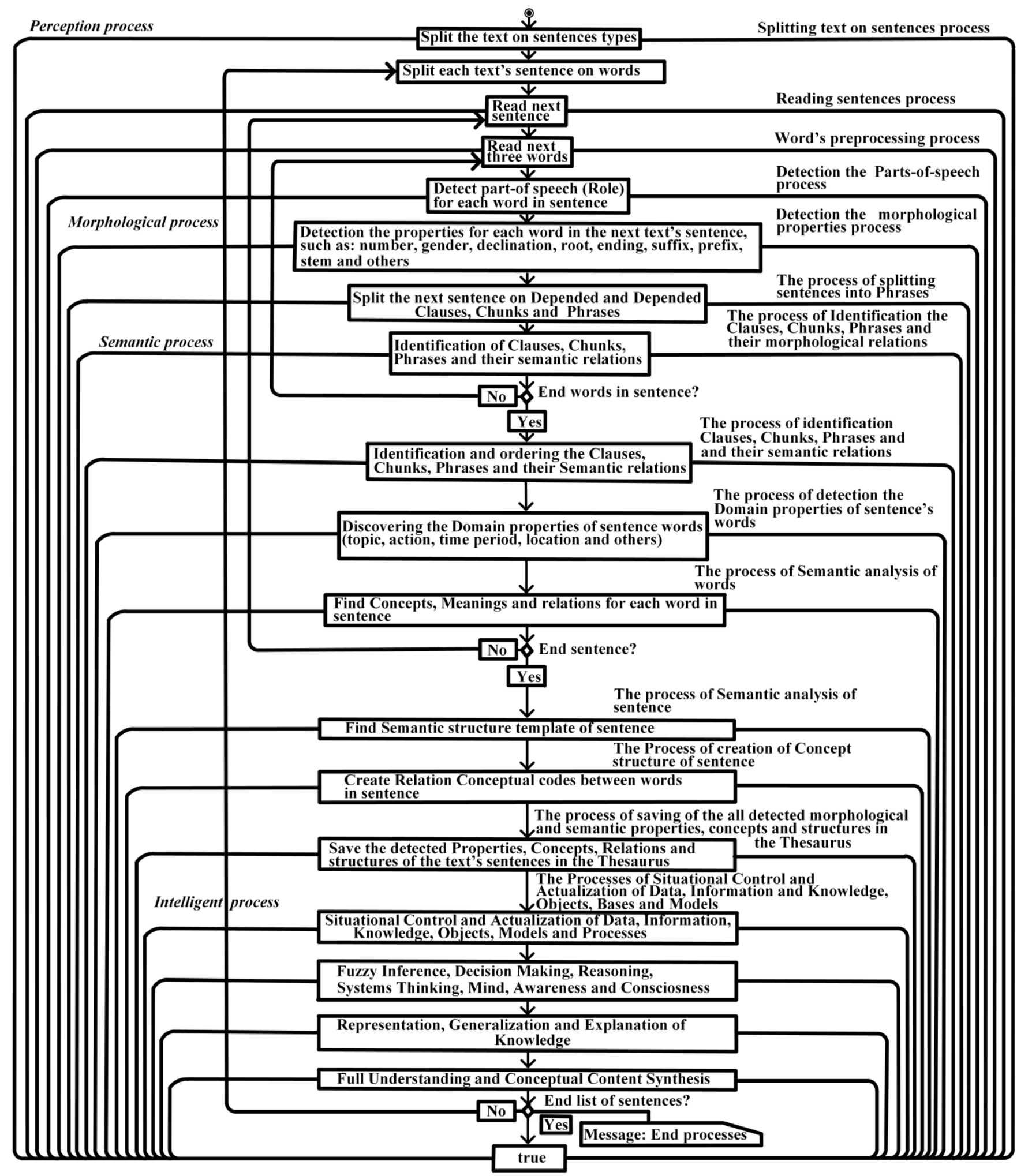

Fig. 6. The Algorithm of Computational Systemic Reasonable Modeling process in Content synthesis from English Texts

The Perception, Structuring, Linking, Storing, Actualization, and Processing stage of the identified data, information, knowledge and objects of languages, images and sounds from reality is carried out by the following steps:
- Detailed extraction of the categories, properties, terms, values from text, images and sound objects.

- Identification, classification, clustering, linking, storing and actualization of these objects in the self-organized computational Neural Network of Semantic Frames. 
- Using the developing procedures for accessing this Network for the extraction, analysis, organization, actualization and storage of syntactical, semantic and intellectual processing of the mentioned objects for the purpose of their computational conscious understanding.

The Systemic Situational Control of Consciousness Modeling stage is represented by the following steps (model (3) and (Fig. 4) and is implemented by computing of meanings of the mentioned objects, storing and their actualizing in the computational Memory by way:

- Forming of the Full State $\mathrm{S}_{\mathrm{i}}$ in the Conscious model $A_{n}^{S}$ and forming its Transformation Operators Q, T and Objectives of Consciousness $F_{n}^{s}$ model (2), (4).

- Mapping the Full State of the $A_{n}^{S}$ model in the Current State of the $B_{n}^{s}$ model by being used mentioned $\alpha_{n}^{\text {Poss }}$ mapping.

- Using of the $\mathrm{I}_{\mathrm{i}}$ Rules for Transformation of the Current State $B_{n}^{s}$ in the New State by operator Q and forming of the mapped New Current State of the $B_{n}^{/ s}$ model.

- Mapping the Current State of the $B_{n}^{J s}$ in the New State of the $A_{n}^{V s}$ by being used mentioned $\beta_{n}^{\text {Poss }}$ mapping.

- Transfer of the $A_{\mathrm{n}}^{\Xi}$ model, being in the past in New Full State of the $A_{n}^{s S}$ model by Operator T.

Wherein, the:

- $\quad \mathrm{x}$ - definite, $\mathrm{w}$ - indefinite and $\mathrm{u}$ - resulting control actions for Situational Control of the consciousness models (1), (2), (3), (4), (Fig. 4).

- $\mathrm{Q}, \mathrm{T}$ - Transformation operators, $\alpha_{n}^{\text {Poss }}$ and $\beta_{n}^{\text {Poss }}$ mapping operators of Situational Control of Data, Information, Knowledge, languages, images and sounds objects, and Consciousness and other mentioned models.

The Systemic Intelligent Processing stage is represented by the following steps of integration and activation of the following process of the Computational Brain System (Fig. 3.):

- Modelling system of Computational Control, Inference and Decision Making.

- Processes of Knowledge Representation, Knowledge.

- Generalization, Knowledge Explanation, Reasoning, Systems Thinking, Cognition, ML, Systemic Mind, Awareness and Consciousness.

\section{CONCLUSION}

The "Consciousness poses the most baffling problems in the science of the mind" [19] and many investigators believe that the problem is intractable.

This paper proposes a solution to the problem of Computational Systemic Consciousness by offered by us new concept, method, model and technology of implementation of the RCUSRUU, based on computational Modeling of the processes of Computational Intelligence by developed by us of the methods of GAI with applying of Fuzzy Logic, Situational Control, Linguistics, Computer Science and other sciences at whole, where all the main components of GAI (Fuzzy Inference, Systems Thinking, Cognition and others, including Consciousness) intellectually computing each other in depending on changes of the state of the system under their uncertainty and in the arisen situation. Wherein, the structure, properties, categories, concepts and values of changing objects in a changing Fuzzy Environment are unknown in advance and their computational perception and cognition are functioned in continuous real time without participation of human intellect and without using the modern technology of ML, based on methods of not always accurate statistical and probabilistic forecasting of results.

In the introduction of our paper, we carried out a scientific analysis and comparison of previous works in the field of the current state of computational consciousness and our approach in this paper to solving the computational consciousness problem for creating GAI systems. As a result of this analysis, we came to the conclusion that these works reflect an outdated approach to the construction of EAI and SAI systems, based on the use of ML technology, which having a sixty year old story and operates on basis of well-known methods of the theory of Probability and Statistics. This approach is difficult to implement, requires huge amounts of statistical data and, due to their probabilistic properties, does not always continuously, situationally and systemically reproduces a relevant and accurate result. Consequently, the use of the modern ML technology to develop systems of Computational Consciousness and systems of GAI is not effective and applicable.

The importance of understanding the need for functioning of systems of Computational Consciousness and GAI under uncertainty lies our definition of "uncertainty" given by us in the introduction to this paper, since the language, sound, visual and other objects surrounding a person are mainly unstructured, the properties and origin of which are not known and insufficient investigated. In this regard, arises necessary in the computational intellectual perception and consciousness of characteristics, states of these objects and the state of their environments in an unknown and arisen situation, when these states and situations change continuously in real time, with what the modern EAI and SAI systems cannot cope.

Our contribution to investigation of Computational Consciousness lies in the fact, that we have developed a new approach to creation of GAI systems, that can computationally perceive, recognize, memorize, think, be aware, self-develop, self-learn and understand linguistic, sound, visual and other objects in in conditions of their change with a Fuzzy Environment, and in unforeseen in situations without the participation of human intelligence in this process and without the use of modern ML technology, based on methods of probability theory and statistics. As a result of the new methods developed by us, the new technology and the new system of Computational Consciousness, functioning under uncertainty with using the main aspects of the System Approach, Fuzzy Logic, Situational Control, Linguistics, and Computer Science, we achieve a constant (sustainable) and unchanged high accuracy in computational Consciousness (based on the using a continuum of possible computational values for membership functions of Fuzzy Logic in the interval $[0,1]$ and other heterogeneous degrees of membership function values (Fig. 1), under conditions of self-adaptation of 
the models, developed by us and of the RCUSRUU to new situation of the surrounding Fuzzy Environment.

In order to resolve this problem, in the paper are proposed the: a) Self-organized Thesaurus, being realized on basis of a Neural Network of fuzzy semantic Frames, built into each other, b) the computational method, a model and a system of Modeling of Systemic Computational Consciousness, based on Models of Systemic Fuzzy Situational Control of Data of the languages, images and sounds objects [2] c) methods of modeling of computational Fuzzy Inference [10], Decision Making [11], Fuzzy Control [11], Reasoning [9], Systems Thinking [12], Cognition, Awareness [18], Mind [8], Presentation, Generalization and Explanation of Knowledge [8],[13], and d) System of the Computational Consciousness, oriented to use in GAI systems as a plugin computationally, autonomously and continuously.

The proposed by us in this paper RCUSRUU is oriented on introduction as a plug in in the ICAS (which can be: robots, spacecrafts, unmanned productions, components of military affairs, design systems, self-learn and self-develop systems, and other), allowing to these systems to inherit the properties of intelligence and an opportunity of functioning computationally, systemically, adaptively, continuously, situationally in real time, under uncertainty and in unknown in advance situations.

\section{References}

[1] B, Khayut, L. Fabri and M, Avikhana, "Intelligent MultiAgent Fuzzy Control System Under Unsetainty," in Journal of Computer Science and Information Technology, vol. 4(18), 2014, pp. 369-380.

[2] B, Khayut and U, Pechersky, "Situational Data Control," Deposited manuscript, VINITI, Moscow, 1987, p. 29.

[3] T. E. Weckowicz, L. A. Bertalanffy, "Pioneer of General Systems Theory", In: University of Alberta; Canada; 2000.

[4] D. A. Pospelov, "Situational Control," Science, 1986, p. 288.

[5] L. A. Zadeh, "Fuzzy Sets," in Information and Control, vol. 8, 1965, pp. 338-359.

[6] L, A. Zadeh, "The Concept of a Linguistic Variable and its Application to Approximate Reasoning," in Information Sciences, vol. 14, pp. 1995, 141-164.

[7] B, Khayut, L. Fabri and M, Avikhana, "Intelligent User Interface in Fuzzy Environment," in International Journal of Artificial Intelligence and Applications, vol. 5(1), 2014, pp. 63-78.

[8] B, Khayut, L. Fabri and M, Avikhana, "Modeling of Computational Systemic Deep Mind Under Uncertainty," in Proc. 8 International Conf. on Complex Adaptive Systems, California, 2016, pp. 253-258.

[9] B, Khayut, L. Fabri and M, Avikhana, "Knowledge Representation, Reasoning and Systems Thinking Under Uncertainty," Proc. 16 International Conference on Computer Modeling and Simulations, Cambridge, 2014, pp. 119-128.

[10]B, Khayut, "Modeling of Fuzzy Logic Inference in Decision-making system," Institute of Mathematics of the
Moldavian Academy of Science," in Modeling Systems, vol. 110, 1989, pp. 134-143.

[11]B, Khayut, L. Fabri and M, Avikhana, "Modeling, Planning, Decision-making and Control in Fuzzy Environment," Proc. International Conference on Complex Adaptive Systems, Philadelphia, pp. 137-143, 2014.

[12] B, Khayut, L. Fabri and M, Avikhana, "Modeling of Intelligent Systems Thinking in Complex Adaptive Systems," in Proc. International Conference on Complex Adaptive Systems, Philadelphia, 2014, pp. 93-100.

[13] Smart Technology World at Intelligent Systems conference, (May 30-31, 2013), Rosewood Hotel, Menlo Park, CA, USA.

[14]D. Gamez, "Progress in machine Consciousness and Cognition," in Journal of Artificial Intelligence Research, vol. 2, 2016, pp. 271-315.

[15] I. Aleksander, "Artificial neuro-consciousness an update: From Neural to Artificial Neural," in Communication, IWANN, vol. 930, 1995, pp. 566-583.

[16] T. Toshiyuki, M. Atsushi, and T. Junichi, "A study of self-awareness in robots," in International Journal of Machine Consciousness, 5(2), 2013, 145-164.

[17] U, Pechersky, and B, Khayut, "Generalization of Knowledge in Decision support systems," in Generalization of Knowledge in Decision support systems," Proc. Conference on Development Computer Facilities, Chisinau, Moldova, 1989, pp. 107-108.

[18]B, Khayut, L. Fabri and M, Avikhana, "A SelfDeveloping Computational System of Full Awareness and Understanding of Reality," in Proc. 4 International Conference on Active Science and Engineering and 29 Modern Artificial Intelligence and Cognitive Science Conference, Spokane, 2018, pp. 6-12.

[19]D, Chalmers, "The problem of consciousness," in Discussions Philosophical, Manizales, Australia, vol. 12(19), 2011.

\section{Creative Commons Attribution License 4.0 (Attribution 4.0 International, CC BY 4.0)}

This article is published under the terms of the Creative Commons Attribution License 4.0 https://creativecommons.org/licenses/by/4.0/deed.en_US 